\title{
Two Dimensional Tidal Current Numerical Model Study on COAL-FIRED Power Project
}

\author{
XiYu Ouyang, a, Meiling Huang ${ }^{1, b}$, Peng Zhao ${ }^{1, c}$ \\ ${ }^{1}$ Key Laboratory of Engineering Sediment of Ministry Communications, Tianjin Research Institute for \\ Water Transportation Engineering; No.2618, Xingang Erhao Road, Tianjin, 300456, China
}

aoyqingjin@163.com, b tj_huangmeiling@163.com, c zhaopeng_tj@163.com

Keywords: Two Dimensional, Tidal Current Numerical Model, COAL-FIRED Power Project

Abstract. Hydrodynamic survey data include tide, tidal current, wind and wave are analyzed near the project location. The two-dimensional tidal current Numerical model is established and are calibrated by the measured hydrographic data to ensure the accuracy of the model. The tidal current model is calculated by nested mesh and unstructured triangular mesh with local refinement is adopted. Tidal level is taken as open boundary. The current fields of pre-project and post-project are simulated through the Numerical model to analyze the influence caused by the water intake and outlet construction.

\section{Introduction}

The two-dimensional tidal current Numerical model is established, and the key parameters of the model are calibrated and validated by the measured hydrographic data to ensure the accuracy of the model. The changes of tidal current field in the sea area around the water intake and wash port are analyzed through the simulation of tidal current Numerical model The Hub II 2x660 MW Coal-Fired Power Plant Project is located in about $56 \mathrm{~km}$ northwest of Karachi district in Pakistan. A location plan is shown in Fig. 1. The project includes water intake, water outlet, sand block and new off-shore coal import jetty, the layout plan is shown in Fig. 2.

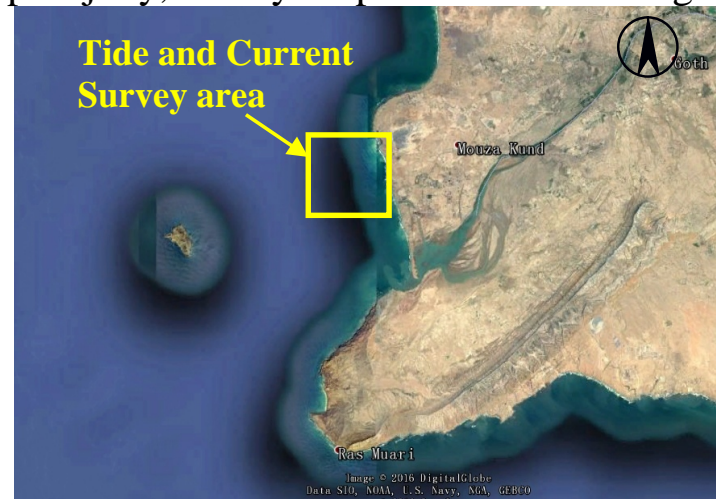

Fig. 1 Location plan of project site

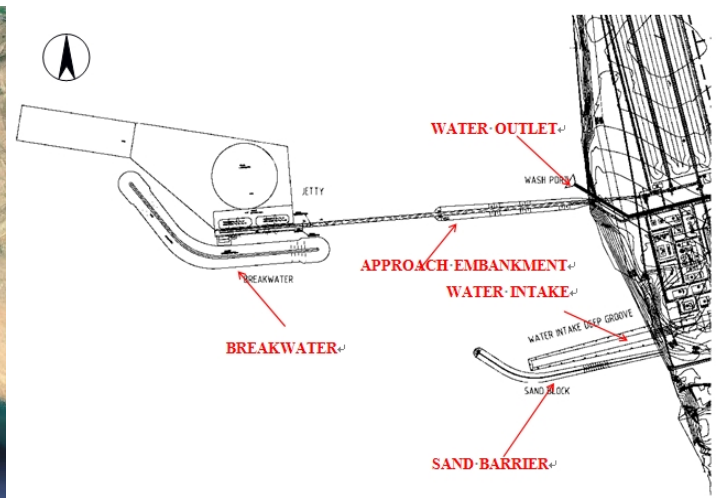

Fig. 2 The layout plan

\section{Natural Conditions}

Tide and Current Conditions. The tide measurement data from June $29^{\text {th }}, 2016$ to $29^{\text {th }}$ July, 2016 is shown in Fig 3. The average tide range is $1.61 \mathrm{~m}$, the highest tide level is $2.88 \mathrm{~m}$ and the lowest is $-0.09 \mathrm{~m}$. The maximum flood tidal range is $2.93 \mathrm{~m}$ and the maximum ebb tidal range is $2.69 \mathrm{~m}$.

Tidal Current Vector graphs of measured data during spring tide and neap tide are shown in Fig 4. On the whole, the survey area shows reciprocating current characteristics and the main current type is mixed current with obvious affection of shallow water constituent. The max depth-averaged velocity during spring tide is $0.17 \sim 0.25 \mathrm{~m} / \mathrm{s}$, during neap tide is $0.18 \sim 0.28 \mathrm{~m} / \mathrm{s}$.

*Corresponding author.

Email address: tj_huangmeiling@163.com. (Meiling Huang). 


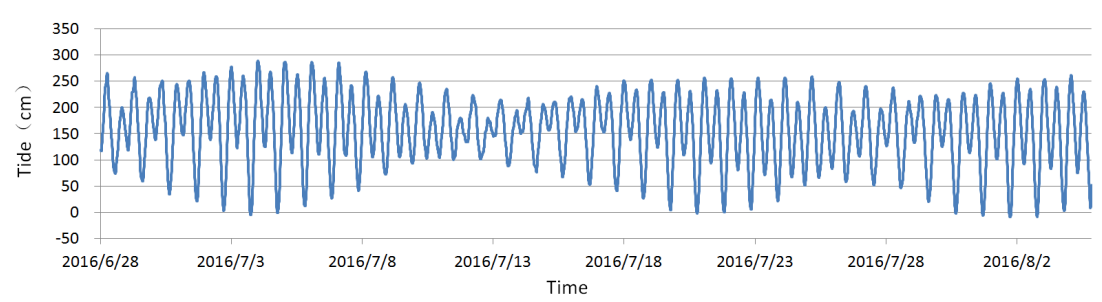

Fig. 3 Tide measurement data graph
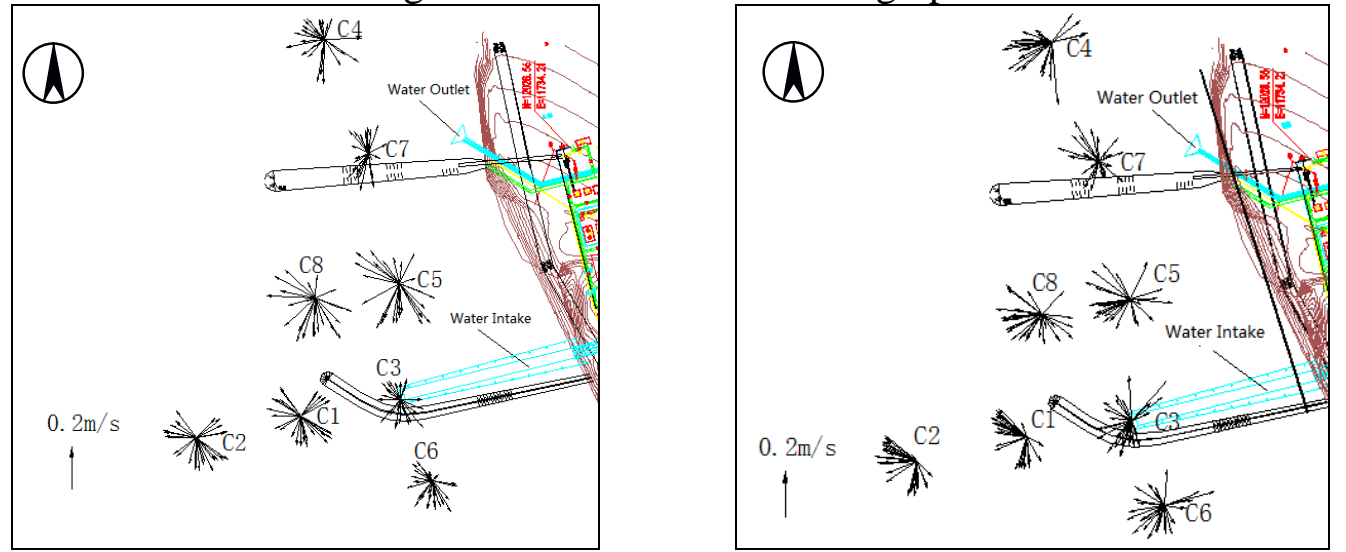

Fig. 4 Tidal Current Vector graphs of measured data (spring tide (L), neap tide(R))

Wind and Wave Conditions. The wind speed and direction are observed from September, $27^{\text {th }}, 2016$ to October, $10^{\text {th }}, 2016$. Wind direction concentrate mainly in SW-W direction. The wind speed is $1.4 \mathrm{~m} / \mathrm{s} \sim 9.2 \mathrm{~m} / \mathrm{s}$ and average value is $4.9 \mathrm{~m} / \mathrm{s}$ during observation.

Wave direction are influenced by the monsoon mainly distributed in S-SW direction which account for $98.63 \%$ and the $\mathrm{SW}$ direction account for $61.48 \%$. The maximum $\mathrm{H}_{\mathrm{s}}$ is $5.38 \mathrm{~m}$ and its average value is $3.13 \mathrm{~m}$. The peak period mainly concentrate in $8 \sim 12 \mathrm{~s}$ which account for $90.12 \%$. The maximum $T_{p}$ is $14.1 \mathrm{~s}$ and its average value is $11.5 \mathrm{~s}$.

\section{Two Dimensional Numerical Model of Tidal Currents}

\section{Governing Equations.}

Continuity equation: $\frac{\partial h}{\partial t}+\frac{\partial h \bar{u}}{\partial x}+\frac{\partial h \bar{v}}{\partial y}=h S$.

Equations of motion

in $\mathrm{X}$ direction:

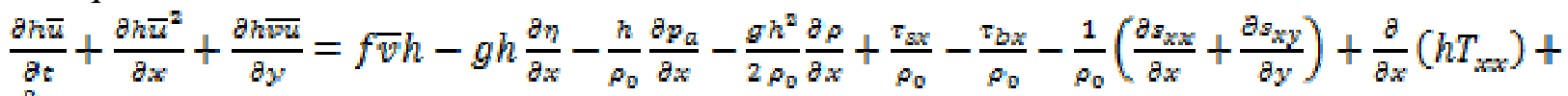
$\frac{\partial}{\partial x}\left(h T_{x y}\right)+h u_{s} S$

Equations

of

direction:

motion in

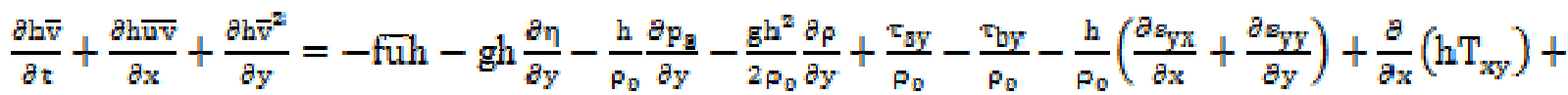
$\frac{\partial}{\partial y}\left(h \mathrm{~T}_{\mathrm{yy}}\right)+h v_{z} \mathrm{~S}$

Where, $t$ is time, $x$ and $y$ are the two coordinate axes in Cartesian coordinates, $\eta$ is water level elevation, $\mathrm{d}$ is water depth, $\mathrm{h}=\eta+d$ is the total depth, $\mathrm{u}$ and $\mathrm{v}$ are the velocity components of $\mathrm{x}$ and $\mathrm{y}, \mathrm{f}=2 \Omega \sin \phi$ is the Coriolis force ( $\phi$ is latitude and $\Omega$ is angle velocity), $\mathrm{g}$ is acceleration of gravity, $\rho$ is density, $\rho_{0}$ is relative density, $v_{z}$ is eddy viscosity, $P_{a}$ is atmosphere pressure, c is material concentration, $D_{x}$ and $D_{y}$ are the diffusion coefficients in $\mathrm{x}$ and y direction, $F$ is linear attenuation coefficient, $S$ is Source of flow ( $u_{z}$ and $v_{z}$ are the corresponding velocity components). Computational Domain and Mesh. The tidal current model is calculated by nested mesh, the scope of large model and small model as show in Fig. 5.The red line shows the large model range and the 
green line shows the small model range. In order to improve the computational efficiency and ensure enough resolution in project area, unstructured triangular mesh with local refinement is adopted. The small model computational mesh is shown in Fig. 6.

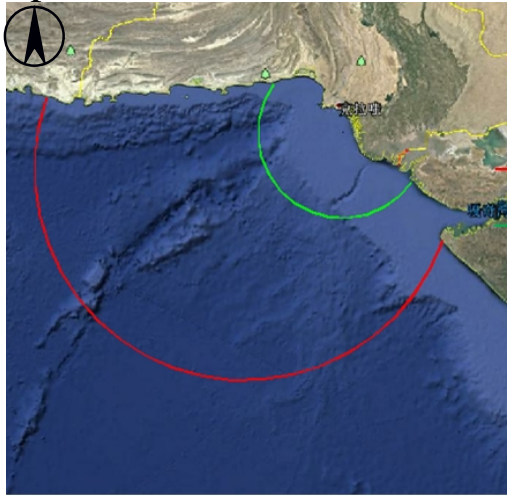

Fig. 5 Nested computational domain

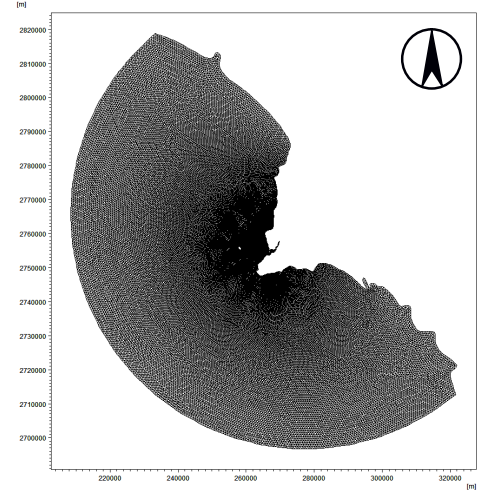

Fig. 6 Small model computational mesh

Model Calibration. Tide level on open boundary for small model is extracted from results of large model. In the tidal current modeling time step ranges from $0.01 \mathrm{~s}$ to $36 \mathrm{~s}$. Wetting and drying method with default parameter values is used to handle moving land boundary. By the calibration eddy viscosity coefficient in Smagorinsky formulation is 0.28 and Manning number is 32 60.

The predicted tide and current will be calibrated with survey data to confirm reliability of this modeling. Comparison curves of measured and simulated tidal level and tidal current in spring tide and neap tide are shown in Fig. 7 Fig. 9.
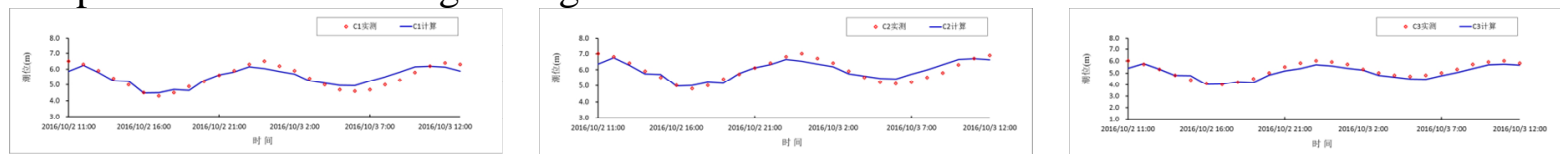

Fig. 7 Tide level comparison during spring and neap tide
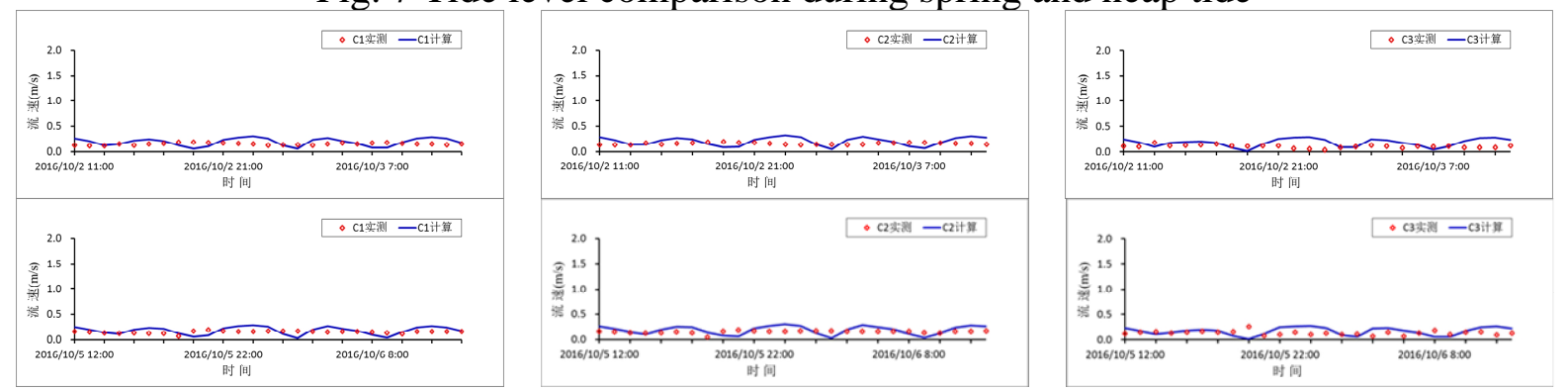

Fig. 8 Current velocity comparison during spring and neap tide
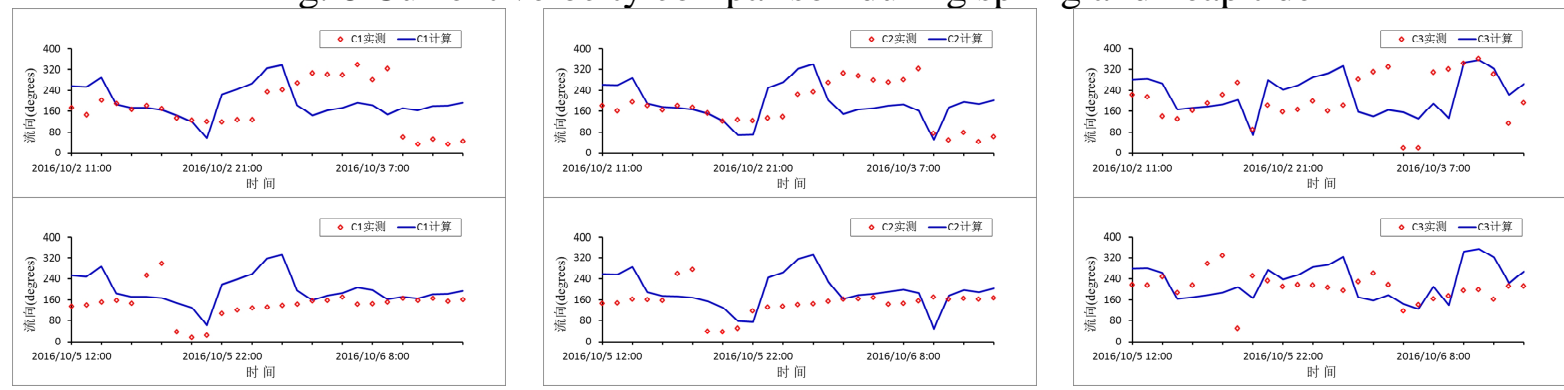

Fig. 9 Current direction comparison during spring and neap tide

\section{Results and Analysis}

The proposed power project is located in the northern Arabian Sea, northwest to the Persian Gulf and the Gulf of Oman, flood and ebb are mainly affected by open sea tidal movements. The sea area near proposed project, influenced by the northern coastline and changes in water depth, the water flow generates circulation during flood and ebb tide, and the water basically flow along the shoreline at the flood peak and ebb peak. 
Fig. 10 Fig. 11 are comparison diagrams of tidal current field before and after project. Before the project, the tidal current fields present the characteristics of periodic reciprocating movement along shore at flood peak and ebb peak. After the project, the flow cross section between breakwater head and leading dike head decreases lead to the velocity increases. In the north side of leading dike, the current direction around water outlet are mainly in $\mathrm{N}$ direction at the flood peak and ebb peak due to the influence of water outlet. Because of the cover of dikes and the flow around the dike head, the velocity significantly decrease and form circulation between leading dike and sand barrier, the circulation is counterclockwise at flood peak and is clockwise at ebb peak. Overall, the velocity decreased in the north side of breakwater and around water intake and wash port. In the north side of sediment-retaining dike and leading dike, the velocity increased due to the influence of water intake and wash port. Around the sediment-retaining dike head and breakwater head, the velocity also increased.
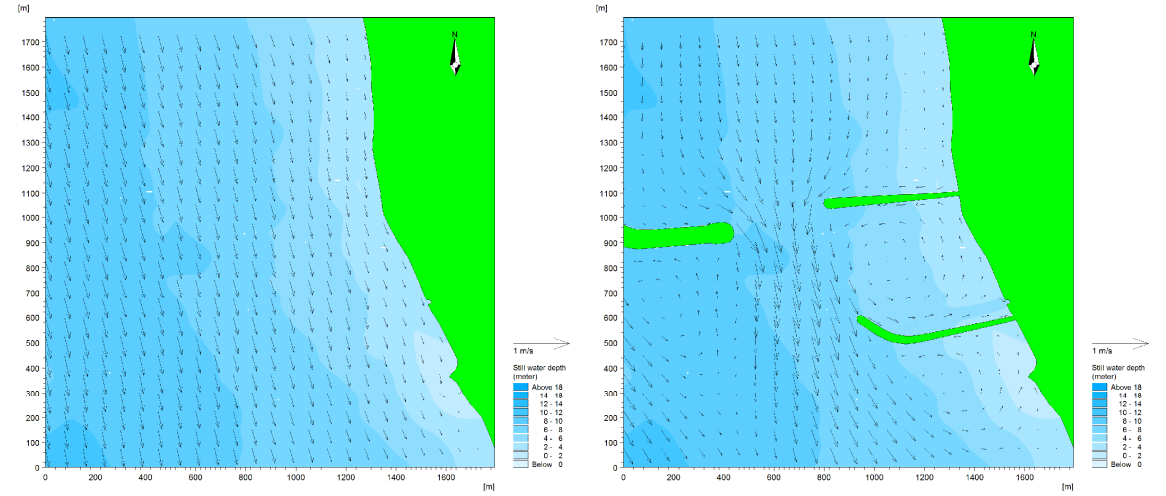

Fig.10 Comparison of the flood current field pre-project(L) and post-project(R) during spring tide
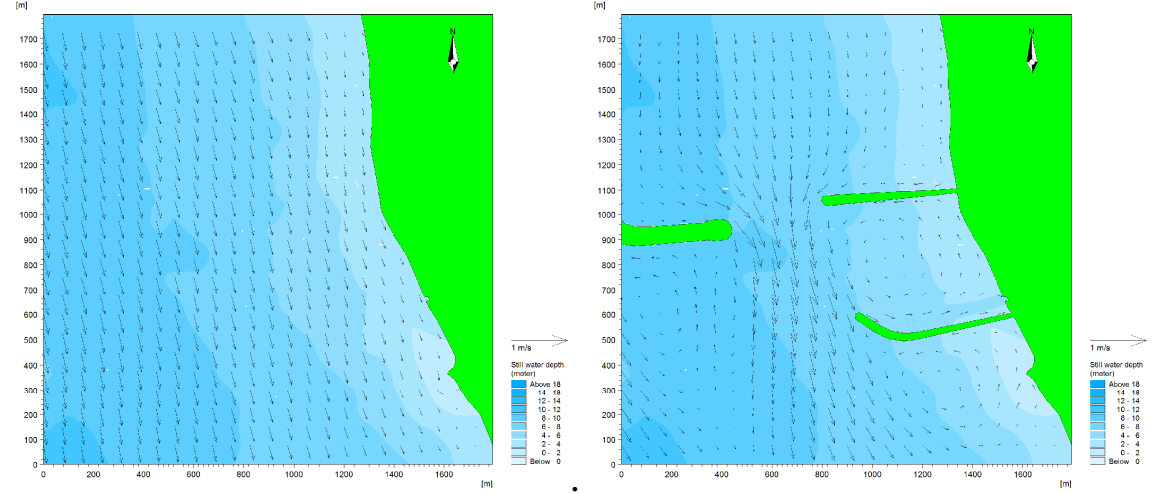

Fig.11 Comparison of the ebb current field pre-project(L) and post-project(R) during spring tide

\section{References}

[1] Study on the Numerical Model of the Current Sediment and Sediment of the New Offshore Coal Import Wharf Project of the Central Bus Hub 2×660MW Coal-fired Power Plant in Pakistan. Tianjin Research Institute for Water Transport Engineering, M.O.T. (2016), in Chinese.

[2] Liu Jiaju: Research and Application of Coastal Sediment Movement (Ocean Publications, Beijing 2009).

[3] Guan Ying. Numerical Simulation and Factor Analysis of Water Intake-Outlet Methods in Power Plant[D]. North China Electric Power University,2012 